\title{
Image reconstruction in optical tomography
}

\author{
SIMON R. ARRIDGE ${ }^{1}$ AND MARTIN SGHWEIGER ${ }^{2}$ \\ ${ }^{1}$ Department of Computer Science, University College London, Gower Street, London WC1E 6BT, UK \\ (s.arridge@cs.ucl.ac.uk) \\ ${ }^{2}$ Department of Medical Physics, University College London, Capper Street, London WC1E 6JA, UK
}

\section{SUMMARY}

Optical tomography is a new medical imaging modality that is at the threshold of realization. A large amount of clinical work has shown the very real benefits that such a method could provide. At the same time a considerable effort has been put into theoretical studies of its probable success. At present there exist gaps between these two realms. In this paper we review some general approaches to inverse problems to set the context for optical tomography, defining both the terms forward problem and inverse problem. An essential requirement is to treat the problem in a nonlinear fashion, by using an iterative method. This in turn requires a convenient method of evaluating the forward problem, and its derivatives and variance. Photon transport models are described and methods for obtaining analytical and numerical solutions for the most commonly used ones are reviewed. The inverse problem is approached by classical gradientbased solution methods. In order to develop practical implementations of these methods, we discuss the important topic of photon measurement density functions, which represent the derivative of the forward problem. We show some results that represent the most complex and realistic simulations of optical tomography yet developed. We suggest, in particular, that both time-resolved, and intensity-modulated systems can reconstruct variations in both optical absorption and scattering, but that unmodulated, nontime-resolved systems are prone to severe artefact. We believe that optical tomography reconstruction methods can now be reliably applied to a wide variety of real clinical data. The expected resolution of the method is poor, meaning that it is unlikely that the type of high-resolution images seen in computed tomography or medical resonance imaging can ever be obtained. Nevertheless we strongly expect the functional nature of these images to have a high degree of clinical significance.

\section{INTRODUGTION}

Optical tomography is a new medical imaging modality that is at the threshold of realization. A large amount of clinical work has shown the very real benefits that such a method could provide (see related papers in these proceedings), and stems from the fact that the relative attenuation of light in tissue at different wavelengths is related to the global concentrations of certain metabolites in their oxygenated and deoxygenated states (Jöbsis 1977; Cope \& Delpy 1988). Currently, there exist commercial instruments for measuring a single detector change in transilluminated radiation and relating this to global, spectroscopic change in these states. However, the development of an optical tomographic imaging system would provide a functional image of local oxygenation. This quest for a tomographic modality follows the course of similar developments that saw the progression of X-ray radiography to X-ray computed tomography (CT), nuclear magnetic resonance spectroscopy (NMRS) to magnetic resonance imaging (MRI), and gamma cameras to single photon emission computed tomography (SPECT) and positron emission tomography (PET). More realistically, optical tomography follows the progression from the impedance camera (Henderson \& Webster 1978) to electrical impedance tomography (EIT).
Due to the very time-consuming nature of data acquisition, considerable effort has been put into theoretical studies of the potential of optical tomography, going so far as to produce simulated images prior to the development of practical data acquisition systems, an order of development possibly unique in the development of medical imaging. However, this has led, unfortunately, to over-extrapolation of the probable realizable potential of the method. In this paper we put forward the approach that we have been developing over several years, based on what we believe to be the most rigorous and accurate analysis of the problem. We suggest that with correct attention to the data acquisition stage, and sufficiently general numerical methods, it is possible to image both absorption and scattering coefficients in objects up to several centimetres in thickness.

This paper is of the nature of a review, but since we have recently published quite detailed reviews of both measurement techniques (Hebden \& Arridge 1997), and modelling and reconstruction (Arridge \& Hebden 1997) some of the more detailed comparison is truncated, with the reader referred to these articles for more details. Instead we summarize our own contributions in this area. In $\S 2$ we review some general approaches to inverse problems to set the context for optical tomography, defining both the terms forward problem and inverse problem, and we review approaches to 
these in $\S 3$ and $\S 4$, respectively. In $\S 5$ we discuss the important topic of photon measurement density functions, which represent the derivative of the forward problem, thus admitting gradient-based solution methods to the inverse problem. In $\S 6$ we show some results that represent the most complex and realistic simulations of optical tomography yet developed. In $\S 7$ we give some final conclusions.

\section{PRINGIPLES OF IMAGE REGONSTRUGTION}

The formation of an image representing one or more internal optical characteristics from a series of boundary measurements is an example of a so-called inverse problem. Specifically, it involves the recovery of the parameters of an appropriate model, such as described in $\S 3$. The forward problem can be stated as follows:

Given a distribution of light sources $\boldsymbol{q}$ on the boundary $\partial \boldsymbol{\Omega}$ of an object $\boldsymbol{\Omega}$, and a distribution of tissue parameters $\boldsymbol{p}$ within $\boldsymbol{\Omega}$, find the resulting measurement set $\boldsymbol{y}$ on $\partial \boldsymbol{\Omega}$.

Solution to the forward problem can be expressed in the form of a general nonlinear forward operator:

$\boldsymbol{y}=\boldsymbol{F}[\boldsymbol{p}(\boldsymbol{r})]$

where $\boldsymbol{F}[\boldsymbol{p}]: \boldsymbol{X}_{\mathrm{p}} \rightarrow \boldsymbol{Y}_{\mathrm{M}}$ is a nonlinear mapping from a space $\boldsymbol{X}_{\mathrm{p}}$ of parameters into a space $\boldsymbol{Y}_{\mathrm{M}}$ of measurements. A single element $\boldsymbol{y} \in \boldsymbol{Y}_{\mathrm{M}}$ represents the observable $M$ for all given source-pair geometries. Similarly, the inverse problem may be stated as follows:

Given a distribution of light sources $\boldsymbol{q}$ and a distribution of measurements $\boldsymbol{y}$ on $\partial \boldsymbol{\Omega}$ derive the tissue parameter distribution $\boldsymbol{p}$ within $\boldsymbol{\Omega}$.

represented by

$\boldsymbol{p}=\boldsymbol{F}^{-1}[\boldsymbol{y}]$,

where $\boldsymbol{F}^{-1}: \boldsymbol{Y}_{\mathrm{M}} \rightarrow \boldsymbol{X}_{\mathrm{p}}$ is a nonlinear mapping from a space $\boldsymbol{Y}_{\mathrm{M}}$ of measurements into a space $\boldsymbol{X}_{\mathrm{p}}$ of parameters. Ideally, we would derive some analytical, or easily computable form for $\boldsymbol{F}^{-1}$, so that an image $\boldsymbol{p}$ could be directly computed from the data given, $\boldsymbol{y}_{d}$, but unfortunately no such solution is currently known. Instead, standard nonlinear solution methods may be applied to equation (1).

Such methods are all predicated, either explicitly or implicitly, on minimizing an appropriate error-norm, which we represent as

$\phi=\left\|\boldsymbol{y}_{d}-\mathrm{F}[\boldsymbol{p}]\right\|$.

The general solution to such problems is the maximum likelihood one, where we seek the solution $\boldsymbol{p}$ that gives rise to a probability distribution function (PDF) $\mathrm{P}[\boldsymbol{y}]$ whose maximum is at $\boldsymbol{y}=\boldsymbol{y}_{d}$. Alternatively we may say that we seek to maximize the conditional probability $\operatorname{Pr}\left[\boldsymbol{y}_{\boldsymbol{d}} \mid \boldsymbol{p}\right]$, where

$\operatorname{Pr}\left[\boldsymbol{y}_{\boldsymbol{d}} \mid \boldsymbol{p}\right] \alpha \exp \left(-\frac{1}{2} \boldsymbol{\phi}\right)$.

In the absence of prior knowledge of the nature of $\mathrm{P}(\boldsymbol{y})$, we seek the maximum a posteriori probability (MAP) estimate. If we assume that there are $D$ measurements, and that each data point $y_{i}$ is corrupted by an error that is independently random with a Gaussian normal distribution with standard deviation $\sigma_{i}$, then the $a$ posteriori probability of the data set $\boldsymbol{y}_{\boldsymbol{d}}$ having been obtained is

$\operatorname{Pr}\left[\boldsymbol{y}_{\boldsymbol{d}} \mid \boldsymbol{p}\right]=\prod_{i=1}^{D}\left\{\frac{1}{\sqrt{2 \pi \sigma_{i}}} \exp \left[-\frac{\left(y_{i}-\mathrm{F}[\boldsymbol{p}]_{i}\right)^{2}}{2 \sigma_{i}^{2}}\right]\right\}$,

which is maximized by the minimization of the errornorm:

$\phi=\sum_{i=1}^{D}\left[\frac{\left(y_{i}-\mathrm{F}[\boldsymbol{p}]_{i}\right)^{2}}{2 \sigma_{i}^{2}}\right]$,

whence the problem reduces to a least-squares (LS) minimization procedure. It is important to note that the correct formulation of the error term needs to take into account not just the data but the standard errors $\sigma_{i}$. In an experimental arrangement we expect to know these errors by appropriate acquisition procedures, but in the development and testing of reconstruction methods we require a method of predicting such errors.

An effective reconstruction procedure thus requires three components: (i) a fast, accurate and flexible forward model; (ii) an estimate of the standard error $\sigma$; and (iii) an efficient minimization scheme.

The forward model that we use is a finite element method (FEM) solution to the diffusion equation for light transport. Its derivation and validation have been presented in several prior publications (Arridge et al. $1992 b, 1993 a, b, 1995 b$; Schweiger et al. $1993 a, b)$. Furthermore it can reliably predict the requisite standard error $\sigma$ for typical measurement types (Arridge et al. 1995). Finally it can be used in a gradient-based minimization technique due to a highly efficient method for computing the derivative of the forward model (Arridge \& Schweiger 1995c). Although alternative approaches have been put forward, we consider the FEM-based method the only generally applicable solution scheme.

\section{THE FORWARD PROBLEM}

\section{(a) Photon transport}

Many medical imaging modalities are governed by the same physical process, represented by the radiative transfer equation (RTE) of atmospheric physics (Chandrasekhar 1950), or the linear transport equation of neutron transport theory (Case \& Zweifel 1967). We write this as:

$$
\begin{aligned}
\left\{\hat{\boldsymbol{s}} \cdot \nabla+\mu_{\mathrm{a}}(\boldsymbol{r})+\mu_{\mathrm{s}}(\boldsymbol{r})+\frac{\partial}{c \partial t}\right\} I(\boldsymbol{r}, \hat{\boldsymbol{s}}, t)= \\
q(\hat{\boldsymbol{s}}, \hat{\boldsymbol{r}}, t)+\mu_{s}(\boldsymbol{r}) \int\left(f\left(\hat{\boldsymbol{s}}^{\prime}, \hat{\boldsymbol{s}}, \boldsymbol{r}\right) I\left(\hat{\boldsymbol{r}}, \hat{\boldsymbol{s}}^{\prime}, t\right)\right) \mathrm{d}^{2} \hat{\boldsymbol{s}}^{\prime},
\end{aligned}
$$

which describes the change of the radiance $I(\boldsymbol{r}, \hat{\boldsymbol{s}}, t)$ at time $t$ at position $\boldsymbol{r}$ into direction $\hat{\boldsymbol{s}}$ within a domain $\boldsymbol{\Omega}$, bounded by a surface $\partial \boldsymbol{\Omega} . q(\boldsymbol{r}, \hat{\boldsymbol{s}}, t)$ is the source term, $\mu_{\mathrm{a}}$ and $\mu_{\mathrm{s}}$ are the absorption and scattering coefficients respectively (dimensions of inverse length), $c$ is the speed of light, and $f\left(\hat{\boldsymbol{s}}^{\prime}, \hat{\boldsymbol{s}}, \boldsymbol{r}\right)$ is the scattering phase function characterizing the intensity of a wave incident in direction $\hat{\boldsymbol{s}}^{\prime}$ scattered into direction $\hat{\boldsymbol{s}}$. 
For example, if $\mu_{\mathrm{s}}$ and $q$ are zero, and the system is assumed to be steady-state, then equation (7) in the steady-state becomes the differential form of the Radon transform for X-ray CT:

$$
\begin{aligned}
\left\{\hat{\boldsymbol{s}} \cdot \nabla+\mu_{\mathrm{a}}(\boldsymbol{r})\right\} I(r, \hat{\boldsymbol{s}})=0 \Rightarrow I(\boldsymbol{b}, \hat{\boldsymbol{s}})= \\
I(\boldsymbol{a}, \hat{\boldsymbol{s}}) \exp -\int_{0}^{1} \mu_{\mathrm{a}}(\boldsymbol{a}+\lambda(\boldsymbol{b}-\boldsymbol{a})) \mathrm{d} \lambda .
\end{aligned}
$$

Similarly if $\mu_{\mathrm{a}}$ and $\mu_{\mathrm{s}}$ are zero and $q$ is non-zero and isotropic, we obtain the Radon transform for SPECT:

$\hat{\boldsymbol{s}} \cdot \nabla I(\boldsymbol{r}, \hat{\boldsymbol{s}})=q(\boldsymbol{r}) \Rightarrow I(\boldsymbol{b}, \hat{\boldsymbol{s}})=\int_{0}^{1} q(\boldsymbol{a}+\lambda(\boldsymbol{b}-\boldsymbol{a})) \mathrm{d} \lambda$. (9)

If both $\mu_{\mathrm{a}}$ and $q$ are non-zero, then the problem becomes the attenuated Radon transform, with the exponential Radon transform representing the case where $\mu_{\mathrm{a}}$ is non-zero and constant.

In optical tomography, scattering is by far the dominant process and Radon transform formulations of the forward problem are not appropriate, except for the tiny fraction of photons that are theoretically available, having undergone no scattering in transit. The discussion of this so-called 'early light' is returned to in $\S 4(a)$. Instead, for practical clinical applications, approximations to the RTE are solved, by considering the expansion of the density $I$, source $q$, and phase function $f$ in spherical harmonics and retaining only a limited number of terms (Lewis 1950; Bremmer 1964). One of the best recent summaries on this topic has been provided by Kaltenbach \& Kaschke (1993) who derive a hierarchy of equations, of which the simplest is the time-dependent diffusion equation:

$$
\begin{aligned}
& \frac{1}{c} \frac{\partial \Phi(\boldsymbol{r}, t)}{\partial t}-\nabla \cdot \Phi(\boldsymbol{r}) \nabla \kappa(\boldsymbol{r}, t)+\mu_{\mathrm{a}}(\boldsymbol{r}) \Phi(\boldsymbol{r}, t)=q_{0}(\boldsymbol{r}, t), \\
& \Gamma(\boldsymbol{r}, t)=-\kappa(\boldsymbol{r}) \nabla \Phi(\boldsymbol{r}, t)
\end{aligned}
$$

where $\Phi$ is the photon density

$$
\Phi(\boldsymbol{r}, t)=\int_{4 \pi} I(\boldsymbol{r}, t, \hat{\boldsymbol{s}}) \mathrm{d}^{2} \hat{\boldsymbol{s}},
$$

and $\Gamma$ is the photon current

$\Gamma(\boldsymbol{r}, t)=\int_{4 \pi} \hat{\boldsymbol{s}} I(\boldsymbol{r}, t, \hat{\boldsymbol{s}}) \mathrm{d}^{2} \hat{\boldsymbol{s}}$

Frequency-domain partial differential equations (PDEs) are easily obtained by Fourier-transforming the time-domain equations. Alternatively they can be derived from first principles by considering the solution to the RTE with an intensity-modulated source. The frequency-domain analogy to equation (10) is given by

$$
-\nabla \kappa(\boldsymbol{r}) \nabla \hat{\Phi}(\boldsymbol{r}, \omega)+\left(\mu_{\mathrm{a}}(\boldsymbol{r})+\frac{\mathrm{i} \omega}{c}\right) \hat{\Phi}(\boldsymbol{r}, \omega)=\hat{Q}_{0}(\boldsymbol{r}, \omega),
$$

where it is to be noted that the frequency is incorporated as a complex attenuation coefficient.

For a more complete treatment of theories and models for light transport, including stochastic methods such as Monte-Carlo and random walk, refer to the review papers by Patterson et al. (1992) and Arridge \& Hebden (1997).

\section{(b) Solution methods for photon transport models}

One of the discussion topics in optical tomography is between the use of exact (analytic) models or those developed computationally. At first sight, analytical expressions would seem preferable, but in practice their range of applicability is very limited, and although bench-top experiments for simple cases bear out their validity, they fail quite badly in complex conditions such as trans-illumination of the neonatal head. However, the conceptual framework of the analytical approach is a useful one in which to set the context of numerical methods.

Analytical solutions for the RTE are scarce and have been obtained for only very simple cases such as onedimensional geometries, e.g. planetary atmospheres. However, for the diffusion approximation, a general methodology that can be adopted is the use of Green's functions - the photon distribution arising when the source is a $\delta$-function. This is of particular interest since the pulsed sources used in optical imaging are often sufficiently close approximations to $\delta$-functions that the Green's function solution gives an accurate model of the time-varying measured intensity.

Green's functions for various homogeneous geometries (slabs, cylinders, spheres) have been published, for both the time- and frequency- domains (Patterson et al. 1989; Arridge et al. 1992a). Eason et al. (1978) provide analytic forms with more complex source conditions including collimated and distributed sources. Recently the analytic form for the Green's function of a sphere embedded in an infinite scattering domain was derived by drawing an electrostatics analogy and matching the gradient of $\Phi$ across the boundary between surfaces (den Outer et al. 1993; Boas et al. 1994; Feng et al. 1995).

For more general geometries, or more complexly inhomogeneous cases, analytic solutions are not available and numerical solutions are the only viable approach. However the concept of a Green's function is still a useful one because it can be derived as the numerical solution resulting from a discrete unit source function. Thence, the mechanism for describing propagators and measurement density functions as described in $\S 5$ can still be applied.

Both the finite difference method (FDM) and the finite element method (FEM) can be used to solve equations such as the RTE and its diffusion approximations. When using FDM for elliptic equations (frequency-domain DE) the multigrid scheme is optimal (Hackbush 1980) and has recently been applied in optical tomography (Pogue et al. 1995). For parabolic equations (time- domain DE) the alternating direction implicit (ADI) scheme is optimal (Ames 1977), provided that the grid is regularly spaced in each of the component $x, y, z$ directions. FDM can also solve the transport equation, provided that the angular integral over scattering directions is discretized ( F. Natterer, personal communication).

The finite element method (FEM) is somewhat more versatile than FDM, especially in regard to complex geometries and for modelling boundary effects, although it suffers from greater computational over- 
Table 1. Absorption and scattering coefficients used in the meshes for figures 2-5

\begin{tabular}{lll}
\hline tissue type & $\mu_{\mathrm{a}} \mathrm{mm}^{-1}$ & $\mu_{\mathrm{s}}^{\prime} \mathrm{mm}^{-1}$ \\
\hline white matter & 0.01 & 1.0 \\
grey matter & 0.04 & 1.55 \\
bone & 0.025 & 2.0 \\
skin & 0.022 & 1.0 \\
\hline
\end{tabular}

head, and cannot be split in the manner of the ADI scheme for finite differencing. An FEM for the transport equation is described by de Oliveira (1986), and the basic application of FEM to solving the diffusion equation has been described by Arridge et al. $(1993 a)$. Its application to the inverse problem was first introduced by Schweiger et al. (1992). Fast methods for deriving measurement operators are described by Arridge \& Schweiger (1995a), and boundary conditions for a diffuse source are discussed by Schweiger et al. (1995). Furthermore, Arridge et al. (1995) provided an analysis wherein the noise properties of a Monte-Carlo model were accurately predicted by a deterministic diffusion approximation.

\section{(c) Applicability of the diffusion approximation}

The diffusion equation is thought to be applicable if the following criteria are considered: (i) the measurements positions are not very close to the source, (ii) the measurement times are not very soon with respect to the input times, and (iii) the absorption coefficient is much less than the scattering coefficient.

The first criterion is easily accounted for by selection of the measurement procedure. The second criterion is often debated - for example, for 'early light' approaches, this assumption is necessarily invalid. Instead the assumption then is that the multiply scattered photons are not involved, and the problem is of Radon type, as discussed in the next section. The third criterion is one which requires further attention. Although it is accepted that media such as skin, bone, brain matter and breast tissue do satisfy this condition (see for example the values in table 1), there also exist non-scattering 'clear layers' in the body, notably cerebral spinal fluid (CSF) in the head. An examination of this effect was carried out by Firbank et al. (1996). A method of compensating for the problem within the context of the diffusion approximation was also presented for a simple slab geometry. In general clinical applications, the effect of clear layers will need to be considered more carefully.

\section{INVERSE SOLUTION METHODS}

\section{(a) Back-projection methods}

The Radon transform is invertible in closed form by a variety of methods, such as resampling in the Fourier domain, filtered back-projection, or back-projection convolution (Natterer 1986). Thus it is attractive to consider an equivalent form for optical CT. If an unscattered component of light could be isolated, a Radon transform in $\left(\mu_{\mathrm{a}}+\mu_{\mathrm{s}}\right)$ could be employed. Back-projection can be represented mathematically by an operator $\boldsymbol{B}: \boldsymbol{Y}_{\mathrm{M}} \rightarrow \boldsymbol{X}_{\mathrm{p}}$, but unless we are lucky, this is not likely to be the same as the required operator $\boldsymbol{F}^{-1}$. Instead a filtration operator is required, either in the space $\boldsymbol{Y}_{\mathrm{M}}$ (filtered back-projection) or in the space $\boldsymbol{X}_{\mathrm{p}}$ (back-projection convolution). The solution is then obtained as

either: $\hat{\boldsymbol{p}}=\boldsymbol{B}\left[\boldsymbol{W}_{\mathrm{Y}}\left[\boldsymbol{y}_{\boldsymbol{d}}\right]\right]$,

or $\quad: \hat{\boldsymbol{p}}=\boldsymbol{W}_{\mathrm{X}}\left[\boldsymbol{B}\left[\boldsymbol{y}_{d}\right]\right]$.

For the Radon transform, forms for $\boldsymbol{W}_{\boldsymbol{X}}$ and $\boldsymbol{W}_{\boldsymbol{Y}}$ can be derived analytically, leading to the great success of $\mathrm{X}$ ray CT. For diffuse light it is commonly suggested (see work reported in Chance \& Alfano $(1993,1995)$ ) that the line integrals in equation (8) could be replaced by integrals over a volume weighted by the photon measurement density functions (see $\S 5$ ), and that back-projection using the same weighting functions replaces the convolution filter $\boldsymbol{W}_{\boldsymbol{X}}$. Various ad hoc backprojection methods have also been postulated and demonstrated (Benaron et al. 1994). However, such formulations have not been proved to solve the inverse problem, and the generalization of these methods to complex-shaped inhomogeneous objects should be treated with caution. In general the form of $\boldsymbol{B}$ and $\boldsymbol{W}_{\boldsymbol{X}}$ can be calculated by FEM but are highly problemdependent.

\section{(b) Perturbation methods}

If we have an estimate $\hat{\boldsymbol{p}}$ that is close to the ideal solution, then its projection $\hat{\boldsymbol{y}}=\boldsymbol{F}[\hat{\boldsymbol{p}}]$ is close to $\boldsymbol{y}$. We can expand equation (1) in a Taylor series:

$\boldsymbol{y}=\boldsymbol{F}[\hat{\boldsymbol{p}}]+\boldsymbol{F}^{\prime}[\hat{\boldsymbol{p}}](\boldsymbol{p}-\hat{\boldsymbol{p}})+(\boldsymbol{p}-\hat{\boldsymbol{p}})^{T} \boldsymbol{F}^{\prime \prime}[\hat{\boldsymbol{p}}](\boldsymbol{p}-\hat{\boldsymbol{p}})+\ldots$

where $\boldsymbol{F}^{\prime}$ and $\boldsymbol{F}^{\prime \prime}$ are the first- and second-order Fréchet derivatives respectively. In the discrete case, these derivatives are over a finite number of dimensions and are represented by matrices $\boldsymbol{F}^{\prime} \rightarrow \boldsymbol{J}$, the Jacobian and $\boldsymbol{F}^{\prime \prime} \rightarrow \boldsymbol{H}$, the Hessian. Putting $\Delta \boldsymbol{y}=(\boldsymbol{y}-\hat{\boldsymbol{y}})$ and $\Delta \boldsymbol{p}=$ $(\boldsymbol{p}-\hat{\boldsymbol{p}})$ leads to:

$\Delta y=J[\hat{p}] \Delta p+\Delta p^{T} H[\hat{p}] \Delta p+\ldots$

Neglecting terms after the first, linear term constitutes the perturbation approach and the problem reduces to inversion of the matrix representation of $\boldsymbol{J}$ at $\hat{\boldsymbol{p}}$. This is therefore a linear problem which may well be illposed, and is amenable to standard matrix inversion methods. Its success is largely dependent on how closely the initial estimate is to the correct solution and how little effect is played by higher-order terms in equation (16).

The majority of reported results use this approach. Without exception they require, either explicitly or implicitly, a difference experiment that measures $\Delta y$ as the difference between two states. This approach provides a means of imaging which is sensitive to changes in 
optical properties, which may be particularly useful for functional imaging of the brain, for example. Graber $e t$ al. (1993) derived $\boldsymbol{J}$ from a Monte-Carlo model and acquired the difference data explicitly by performing an experiment with and without embedded absorbers. Arridge et al. (1991) performed a similar procedure with an analytical kernel and with experimental data derived by a differencing experiment. O'Leary et al. (1995) also used an analytical kernel, but derived the difference data implicitly by using two sources and subtracting the measured values. The investigators made the assumption that the resulting difference is an approximation to that obtained in an actual difference experiment, which is reasonable given that the image was of a localized perturbation and the sources and detectors were relatively far away.

\section{(c) Nonlinear optimization methods}

The nonlinear approach seeks to repeatedly update an estimate of the solution, until the value of the errornorm evaluated at each trial solution has reached an acceptable minimum. It is thus essential to have a fast forward model. A seminal paper by Singer et al. (1990) introduced this approach using a Markov random field model on a discrete lattice to recover absorption and directional scattering parameters. The approach employed by Arridge et al. $(1992 b, 1993 b)$ uses FEM for the forward model and a Newton-Raphson scheme to progress towards the minimization of a least-squared error-norm iteratively. Levenburg-Marquardt conditioning, together with Tikhonov and PhillipsTwomey regularization were used to control the stability of the solution. Recently the same method was applied by Jiang et al. (1995) to frequency-domain data.

\section{DERIVATION OF THE MODEL DERIVATIVE}

Both the perturbation approach and the nonlinear approach make use of the Fréchet derivative of $\boldsymbol{F}$. In the continuous case this is a mapping $\boldsymbol{F}^{\prime}[\boldsymbol{p}]: \boldsymbol{X}_{\mathrm{p}} \rightarrow$ $\left(\boldsymbol{Y}_{\mathrm{M}} \times \boldsymbol{X}_{\mathrm{p}}\right)$, and in the discrete case, the Jacobian matrix $J$. To gain a physical insight into the meaning of the Jacobian, suppose we have an experiment that measures property $M$ at a set of source-measurement pairs $\left(\xi_{1}, \xi_{2}, \ldots, \xi_{N}\right) \times\left(\zeta_{1}, \zeta_{2}, \ldots, \zeta_{S}\right)$ and we divide the domain $\Omega$ into $L$ non-overlapping elemental regions $\left(\tau_{1}\left(\boldsymbol{r}^{\prime}\right), \tau_{\mathbf{2}}\left(\boldsymbol{r}^{\prime}\right), \ldots \tau_{L}\left(\boldsymbol{r}^{\prime}\right)\right)$ such that $\Omega=\cup_{i=l}^{1} \tau_{i}(\boldsymbol{r})$, then $J$ can be represented:

$\left[\begin{array}{c}\Delta y\left(\xi_{1}, \zeta_{1}\right) \\ \Delta y\left(\xi_{1}, \zeta_{2}\right) \\ \cdot \\ \cdot \\ \Delta y\left(\xi_{i}, \zeta_{k}\right) \\ \cdot \\ \cdot \\ \Delta y\left(\xi_{S}, \zeta_{N}\right)\end{array}\right]=\left(\begin{array}{ccccc}J_{p}^{(M)}\left(\xi_{1}, \zeta_{1} ; \boldsymbol{r}_{1}^{\prime}\right) & \ldots & J_{p}^{(M)}\left(\xi_{1}, \zeta_{1} ; \boldsymbol{r}_{j}^{\prime}\right) & \ldots & J_{p}^{(M)}\left(\xi_{1}, \zeta_{1} ; \boldsymbol{r}_{L}^{\prime}\right) \\ J_{p}^{(M)}\left(\xi_{2}, \zeta_{1} ; \boldsymbol{r}_{1}^{\prime}\right) & \ldots & J_{p}^{(M)}\left(\xi_{2}, \zeta_{1} ; \boldsymbol{r}_{j}^{\prime}\right) & \ldots & J_{p}^{(M)}\left(\xi_{2}, \zeta_{1} ; \boldsymbol{r}_{L}^{\prime}\right) \\ \cdot & \ldots & \cdot & \ldots & \cdot \\ \cdot & \ldots & \cdot & \ldots & \cdot \\ J_{p}^{(M)}\left(\xi_{k}, \zeta_{i} ; \boldsymbol{r}_{1}^{\prime}\right) & \ldots & J_{p}^{(M)}\left(\xi_{k}, \zeta_{i} ; \boldsymbol{r}_{j}^{\prime}\right) & \ldots & J_{p}^{(M)}\left(\xi_{k}, \zeta_{i} ; \boldsymbol{r}_{L}^{\prime}\right) \\ \cdot & \ldots & \cdot & \ldots & \cdot \\ \cdot & \ldots & \cdot & \ldots & \cdot \\ J_{p}^{(M)}\left(\xi_{N}, \zeta_{S} ; \boldsymbol{r}_{1}^{\prime}\right) & \ldots & J_{p}^{(M)}\left(\xi_{N}, \zeta_{S} ; \boldsymbol{r}_{j}^{\prime}\right) & \ldots & J_{p}^{(M)}\left(\xi_{N}, \zeta_{S} ; \boldsymbol{r}_{L}^{\prime}\right)\end{array}\right)\left[\begin{array}{c}\Delta \boldsymbol{p}\left(\boldsymbol{r}_{1}^{\prime}\right) \\ \cdot \\ \cdot \\ \Delta \boldsymbol{p}\left(\boldsymbol{r}_{j}^{\prime}\right) \\ \cdot \\ \cdot \\ \Delta \boldsymbol{p}\left(\boldsymbol{r}_{L}^{\prime}\right)\end{array}\right]$

The entries in the Jacobian represent the sensitivity of a particular measurement at a detector $\xi_{k}$, from a source $\zeta_{j}$ to changes in the image parameters $\boldsymbol{p}\left(\boldsymbol{r}_{i}\right)$. Considered as matrix columns, they represent the perturbation in data due to a point inhomogeneity. Considered as matrix rows, they represent the "photon measurement density functions' (PMDFs) as recently introduced by Arridge (1995) and Arridge \& Schweiger $(1995 c)$.

Various methods exist to derive the basic perturbation equations. Arridge et al. (1991) used a linear perturbation method to derive the change in intensity in both the Fourier and the temporal domain, for both absorption and scattering changes. The absorption term for the temporal case was derived by Schotland $e t$ al. (1993) by taking the first term in the Feynman path integral expression for the Hamiltonian operator. Feng et al. (1995) derived the absorption term for the steadystate case by taking the limiting value of the exact perturbed intensity of a spherical inhomogeneity as the radius of the inhomogeneity reduced to zero, and Sevick et al. (1994) derived the same result using a Monte-Carlo argument. O'Leary et al. (1995) noted the similarity of the frequency-domain case to the Born approximation for a scattered wave.

Alternatively, instead of considering changes in intensity, changes in some transformation of the intensity can be used to derive the Jacobian. Arridge $e t$ al. $(1992 b)$ compared reconstruction from intensity and $\log$ intensity measurements and found the latter provided considerable improvement. This was later confirmed by O'Leary et al. (1995) who pointed out that the logarithmic intensity is equivalent to the Rytov approximation whereas absolute intensity is the Born approximation. More generally we can consider the logarithmic transformation as one example of a measurement operator or postprocessing transformation on the obtained data $\Gamma(t)$. The general treatment of any kind of measurement operation is given by Arridge (1995). In particular we consider normalized integral transforms of the time-varying intensity:

$$
\begin{array}{lll}
\text { Mellin } & \left\langle t^{n}\right\rangle=\frac{1}{E} \int t^{n} \Gamma(t) \mathrm{d} t, \\
\text { Laplace } & \mathscr{L}(s)=\frac{1}{E} \int e^{-s t} \Gamma(t) \mathrm{d} t, \\
\text { Modulation } & A(\omega)=\frac{1}{E}|\hat{\Gamma}(\omega)|, \\
\text { Phase } & \Psi(\omega)=\operatorname{Arg}[\hat{\Gamma}(\omega)],
\end{array}
$$



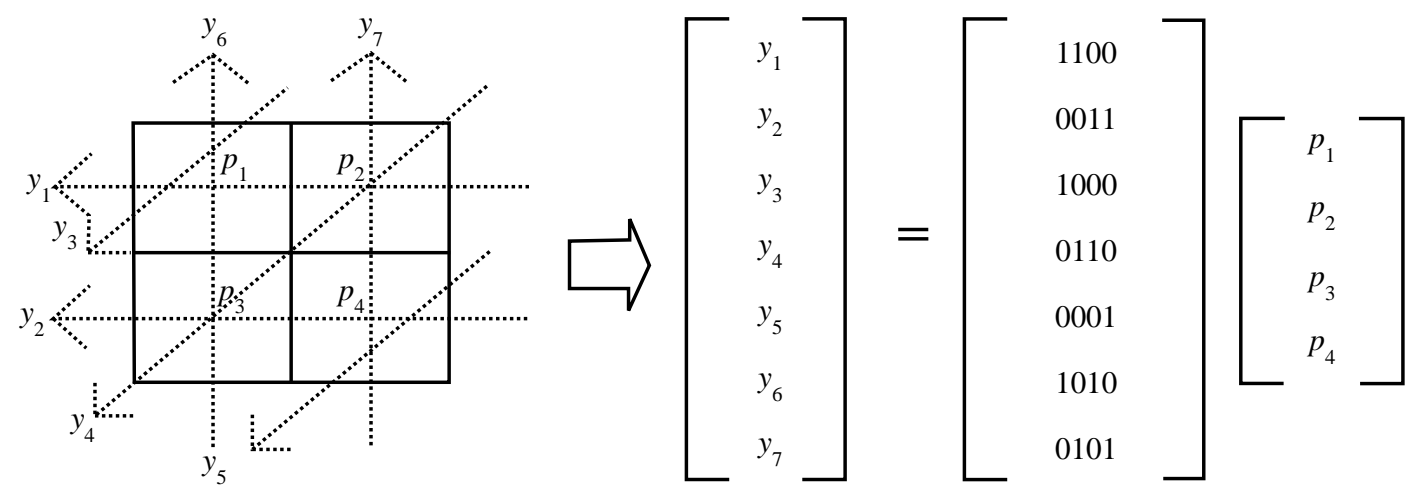

Figure 1. The Radon transform for X-ray CT represented as a matrix problem. In this idealized case, only four pixels and seven measurements are shown, with each measurement being a sum over the equally weighted pixel values intersected by a ray from source to detector.

where $E$ is the integral of $\Gamma(t)$. The advantage of these transforms is that they overcome measurement difficulties in determining absolute intensity across a wide dynamic range. Furthermore, as shown in $\S 6$, examination of the PMDFs for different measurement types reveals dramatic differences in the possibilities for reconstructing parameters far from the source or detector.

For the Radon transform as given in equation (8) where $M$ is the logarithm of the relative attenuation, and $\boldsymbol{p}$ is $\mu_{\mathrm{a}}(\boldsymbol{r})$, the Fréchet derivative is a constant. If $\boldsymbol{p}(\boldsymbol{r})$ is sampled on a pixel basis then each row of $J$ is just the set of pixels intersected by the straight line from source to detector as shown in figure 1. It is important to note that for a transport model based on equation (10), $\boldsymbol{p}(\boldsymbol{r})$ needs to be considered as a vector $\left(\mu_{\mathrm{a}}(\boldsymbol{r}), \kappa(\boldsymbol{r})\right)$. We have shown previously that non-timeresolved methods are unable to distinguish between the two images simultaneously (Arridge et al. 1993b), which is a serious shortcoming of some approaches that only attempt reconstruction of $\mu_{\mathrm{a}}(\boldsymbol{r})$.

Under the assumption of equation (10), the Fréchet derivative for simple geometries is analytically derivable. For the case where $\boldsymbol{p}$ is absorption coefficient $\mu_{\mathrm{a}}$, the time-dependent Fréchet derivative in an homogeneous infinite space is

$$
\begin{aligned}
& J_{\alpha, \text { inf }}^{(\Gamma)}\left(\xi, \zeta, t ; \boldsymbol{r}^{\prime}\right)= \\
& {\left[\left(\hat{\boldsymbol{s}}(\xi)\left(\xi-\boldsymbol{r}^{\prime}\right)\right] S^{(I)}\left(\left|\xi-\boldsymbol{r}^{\prime}\right|,\left|\boldsymbol{r}^{\prime}-\zeta\right|, t\right),\right.}
\end{aligned}
$$

where

$S^{(\Gamma)}(x, y, t)=\frac{1}{2(2 \pi)^{3}}\left(\frac{1}{y^{3}}+\frac{(x+y)^{2}}{2 \kappa t x y^{2}}\right) f(x+y, t)$,

and

$f(x, t)=(2 \kappa t)^{-3 / 2} \exp \left(-\gamma t-\frac{x^{2}}{4 \kappa t}\right)$.

In the frequency domain we require just the Fourier transform of $S^{(I)}(x, y, t)$ given by

$\hat{S}^{(\Gamma)}(x, y, \omega)=\frac{1}{4(2 \pi)^{3} \kappa} \frac{1+\sigma y}{x y^{2}} \exp [-\sigma(x+y)]$,

with $\sigma=\left(\left(\mu_{\mathrm{a}} \mathrm{c}+\mathrm{i} \omega\right) / \kappa\right)^{1 / 2}$. Determination of these functional forms might suggest their use in a backprojection operator, but unfortunately the shape of the functions changes in a very nonlinear manner when inhomogeneous, or geometrically complex measurement domains are considered. Instead, these generalizations can be accounted for within the FEM framework using reciprocity relationships described in Arridge \& Schweiger $(1995 c)$.

\section{RESULTS}

To examine the potential for optical tomography in the neonatal brain, we carried out simulations on a model generated from an MRI head scan. To create a two-dimensional mesh based on an MRI image of the head, an automated mesh generation routine was developed, based on an adaptive mesh generation method (Peraire et al. 1987), and has been extended to take into account the internal boundaries of tissue types. First an edge-detection routine was applied to the image to calculate the outline of the head and all internal structures of interest as a series of closed polygons. The generation algorithm then proceeds by filling each area by triangular elements, starting from the polygonal boundaries. The absorption and scattering coefficients of the mesh elements are then set to the values appropriate for the identified tissue type. The mesh used here is shown in figure 2. The optical parameters of each element are indicated as greyscales, left $\mu_{\mathrm{a}}$ and right $\mu_{\mathrm{s}}^{\prime}$. The mesh consists of 29639 elements and 15025 nodes. We distinguish four different tissue types: skin, bone, grey and white brain matter, with values given in table 1 . The mesh may be scaled in size for simulation of different clinical situations. For simulating neonatal heads we use a sagittal diameter (posterior-anterior distance) of $10 \mathrm{~cm}$.

In figures 3 and 4 we show examples of PMDFs for absorption perturbations, for integrated intensity measurements $(E)$ and mean time $\langle t\rangle$ respectively. The grey-scale intensity displayed in the figures indicates the relative sensitivity of the measurement to a change in attenuation coefficient. The most striking feature in each case is their complexity - a simple Radon transform approximation would yield a straight line from source to detector. The second most notable feature is that the integrated intensity measurement is dependent almost entirely on the features of the image directly under the source and detector. Almost no 

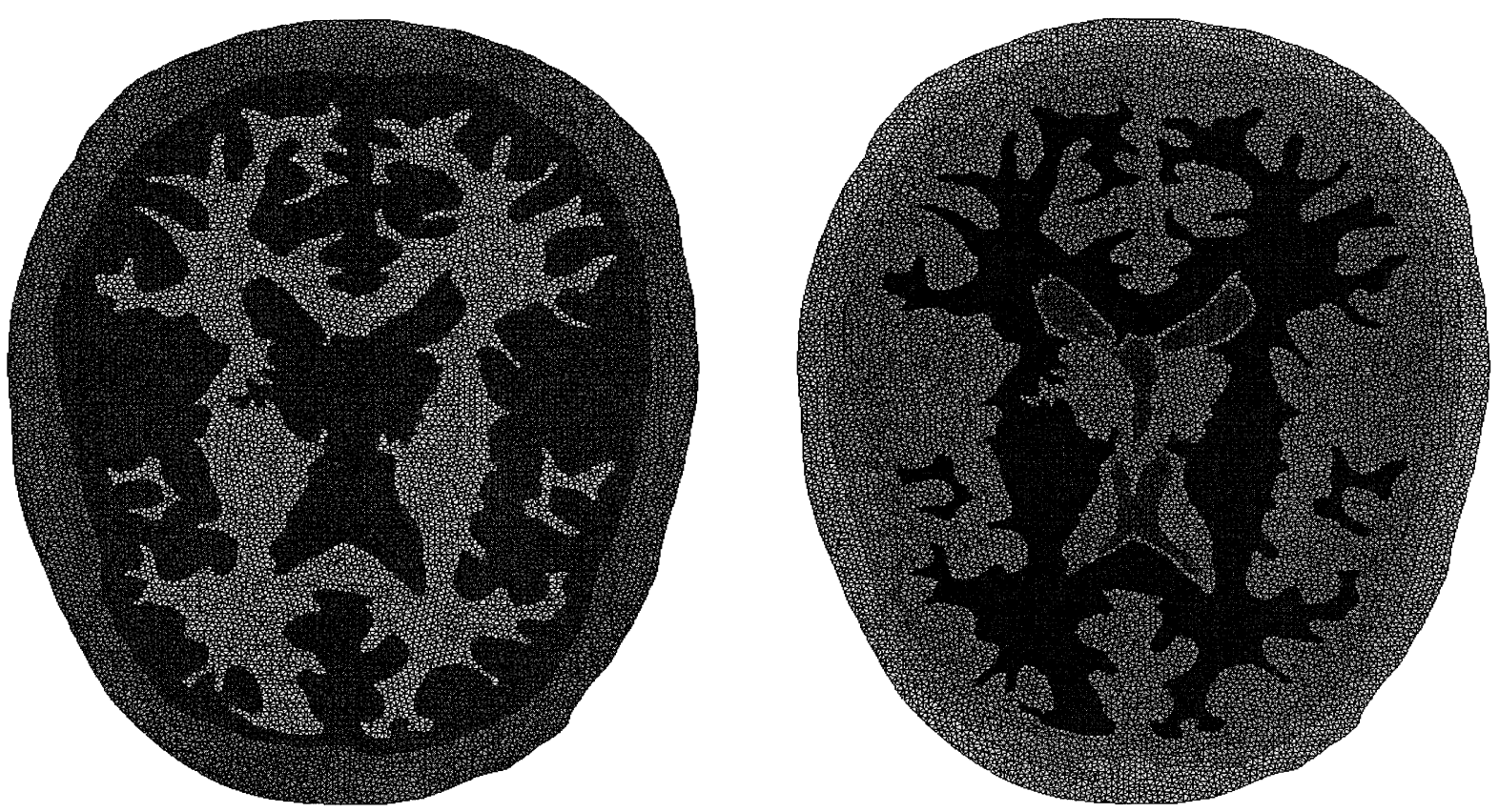

Figure 2. A finite element mesh derived directly from an MRI scan. The decomposition into four regions was achieved automatically using a contour tracking algorithm. Each element in the mesh contains a value for absorption coefficient $\mu_{\mathrm{a}}$ and reduced scattering coefficient $\mu_{\mathrm{s}}^{\prime}$ taken from published estimates.

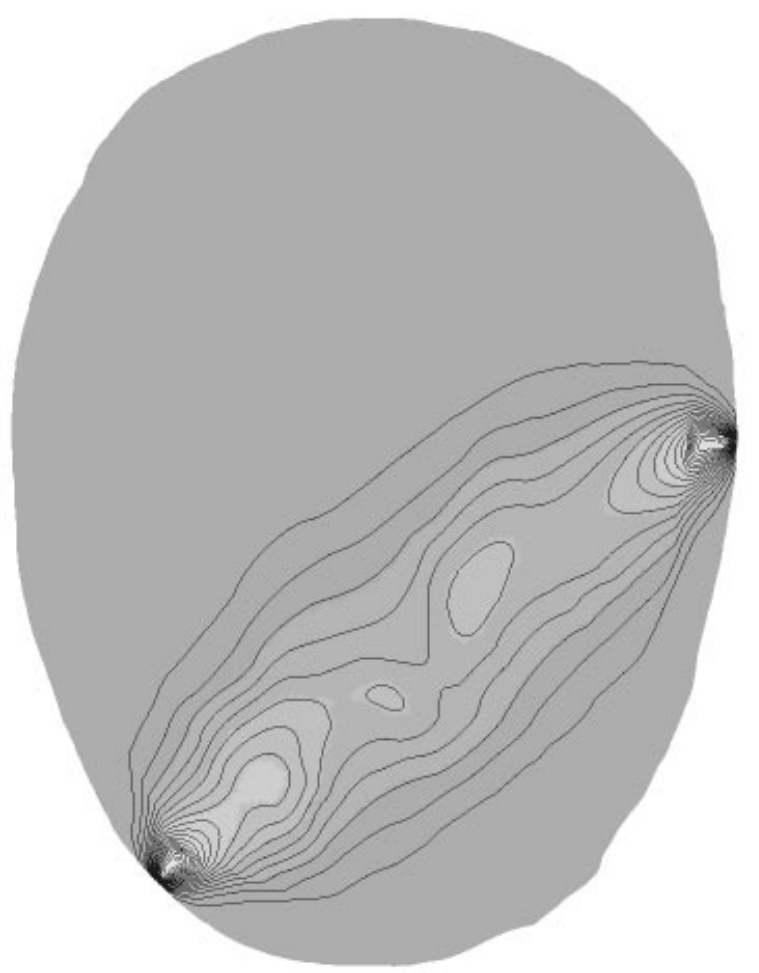

Figure 3. The photon measurement density function (PMDF) corresponding to integrated intensity measurement at a single source detector pair, and absorption change only.

information is derived from the interior of the brain. In contrast the mean time measurement penetrates deeply into the brain and samples more strongly from the interior.

It is important to note that these simulations still assume the validity of the diffusion approximation. The marked difference from the simple forms of equations (20) and (22) indicate the inadvisability of

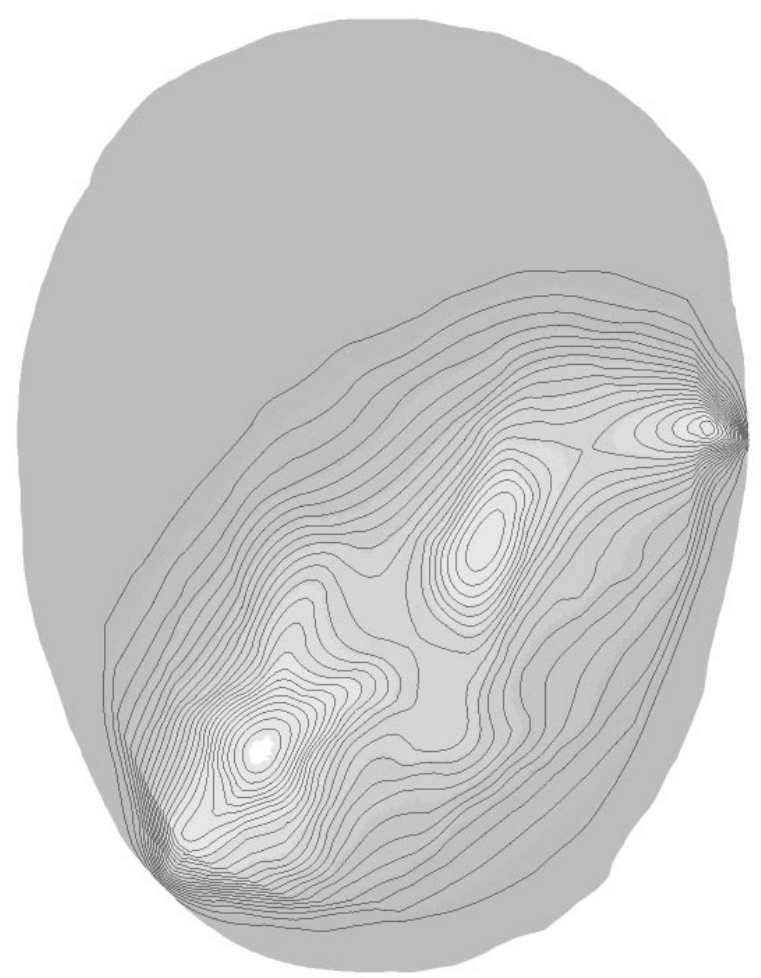

Figure 4. The photon measurement density function (PMDF) corresponding to mean time measurement at the same single source detector pair as in figure 3 , and absorption change only.

straightforward back-projection reconstruction algorithms, even given this restriction. The behaviour of the PMDFs will become even more complex in a fully three-dimensional model and when the effect of the CSF layer is taken into account. Thus these results are meant to indicate an incremental step in generality 


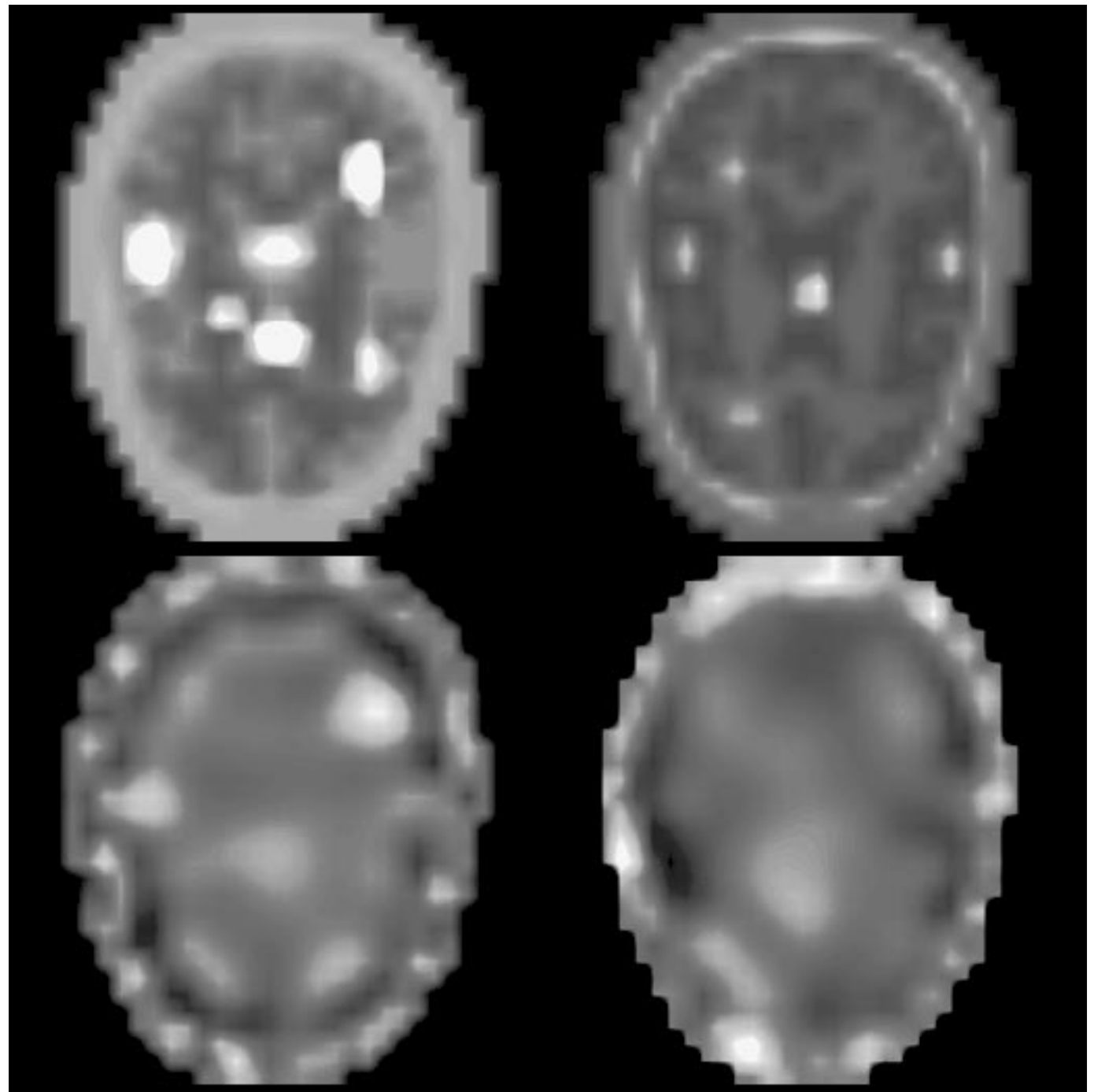

Figure 5. Simultaneous reconstruction of absorption $\left(\mu_{\mathrm{a}}\right.$, left $)$ and scattering $\left(\mu_{\mathrm{s}}^{\prime}\right.$, right) distributions from a homogeneous starting condition. The top row shows the target images, obtained by taking the mesh shown in figure 2, with added localized perturbations, and resampling into a pixel basis. The bottom row shows the reconstructed images, obtained from mean time $\langle t\rangle$ and Laplace-transformed data (transform value $s=0.001 \mathrm{ps}^{-1}$ ).

without yet claiming to solve the full clinical problem of optical imaging in the brain.

We generated data for 32 equally spaced data points and 32 interleaved measurement sites, giving 1024 measurements per data type. Included in the mesh were several small groups of elements with a contrast of between $50 \%$ and $200 \%$ in $\mu_{\mathrm{a}}$ and $\mu_{\mathrm{s}}$ representing a highly localized increase in absorption. We used single samples of both the Mellin and Laplace transforms as the data types and attempted reconstruction assuming only a homogeneous distribution of $\mu_{\mathrm{a}}(\boldsymbol{r})=$ $0.022 \mathrm{~mm}^{-1}$ and a homogeneous distribution of $\mu_{\mathrm{s}}^{\prime}(\boldsymbol{r})=1.0 \mathrm{~mm}^{-1}$. These values equal the parameters representing skin in the model, since it is possible that surface characteristics can be determined by other means. In figure 5 we show the target (idealized) and obtained reconstructions of both $\mu_{\mathrm{a}}$ and $\mu_{\mathrm{s}}$. Despite considerable artefact, especially in the boundary as is to be expected, the algorithm has succeeded in identifying several of the absorbtion anomalies, as well as disambiguating absorption and scatter to a surprisingly high degree. It can be seen that the resolution of the scatter reconstruction is much less than the absorption reconstruction. These images represent the best likely to be obtainable in real clinical conditions.

\section{GONGLUSIONS}

The optical tomography problem presents some interesting difficulties for both experimental and theoretical work. This paper has attempted an overview of the theoretical problems for image reconstruction. To do this it is essential to consider the nonlinear nature of the forward problem. A wide variety of methods are available both for the forward and inverse problems. An essential requirement is to treat the problem in a nonlinear fashion, by using an iterative method. This in turn requires a convenient method of evaluating the forward problem, and its derivatives and variance. All of these requirements are met by the finite element approach, and we believe that optical tomography reconstruction methods can now be reliably applied to a wide variety of real clinical data.

The expected resolution of the method is poorresolving up to $5 \mathrm{~mm}$ objects to a depth of 3-4 cm, such resolution decreasing with increasing depth. Incremen- 
tal improvement may come from the use of auxiliary prior knowledge, but it is unlikely that the type of highresolution images seen in CT or MRI can ever be obtained. Nevertheless we strongly expect the functional nature of these images to have a high degree of clinical significance.

Support has been generously provided by Action Research, the Wellcome Trust and Hamamatsu Photonics.

\section{REFERENGES}

Ames, W. F. 1977 Numerical methods for partial differential equations, 2nd edn. New York: Academic Press.

Arridge, S. R. 1995 Photon measurement density functions. 1. Analytic forms. Appl. Opt. 34, 7395-7409.

Arridge, S. R. \& Hebden, J. 1997 Optical imaging in medicine II: modelling and reconstruction. Phys. Med. Biol. 42 (5), 841-853.

Arridge, S. R. \& Schweiger, M. 1995 a Direct calculation of the moments of the distribution of photon time of flight in tissue, using a finite element method. Appl. Opt. 34, 2683-2687.

Arridge, S. R. \& Schweiger, M. $1995 b$ Sensitivity to prior knowledge in optical tomographic reconstruction. Proc. SPIE 2389, 378-388.

Arridge, S. R. \& Schweiger, M. 1995c Photon measurement density functions. Part II : finite element method calculations. Appl. Opt. 34, 8026-8037.

Arridge, S. R., Cope, M. \& Delpy, D. T. 1992 a Theoretical basis for the determination of optical pathlengths in tissue: temporal and frequency analysis. Phys. Med. Biol. 37, 1531-1560.

Arridge, S. R., Hiraoka, M. \& Schweiger, M. 1995 Statistical basis for the determination of optical pathlength in tissue. Phys. Med. Biol. 40, 1539-1558.

Arridge, S. R., Schweiger, M. \& Delpy, D. T. $1992 b$ Iterative reconstruction of near infrared absorption images. Proc. SPIE 1767, 372-383.

Arridge, S. R., Schweiger, M., Hiraoka, M. \& Delpy, D. T. $1993 a$ A finite element approach to modelling photon transport in tissue. Med. Phys. 20, 299-309.

Arridge, S. R., Schweiger, M., Hiraoka, M. \& Delpy, D. T. $1993 b$ Performance of an iterative reconstruction algorithm for near infrared absorption imaging. Proc. SPIE 1888, 360-371.

Arridge, S. R., Van der Zee, P., Cope, M. \& Delpy, D. T. 1991 Reconstruction methods for infrared absorption imaging. Proc. SPIE 1431, 204-215.

Benaron, D. A., Ho, D. C., Spilman, S. D., Van Houten, J. P. \& Stevenson, D. K. 1994 Non-recursive linear algorithms for optical imaging in diffusive media. In Advances in experimental medical biology. Oxygen transport to tissue XVI. New York: Plenum Press.

Boas, D. A., O'Leary, M. A., Chance, B. \& Yodh, A. G. 1994 Scattering of diffuse photon density waves by spherical inhomogeneities within turbid media: analytic solutions and applications. Proc. Natn. Acad. Sci. USA 91, 4887.

Bremmer, H. 1964 Random volume scattering. Rad. Sci. J. Res. 680, 967-981.

Case, M. C. \& Zweifel, P. F. 1967 Linear transport theory. New York: Addison-Wesley.

Chance, B. \& Alfano, R. R. (eds) 1993 Photon migration and imaging in random media and tissues. Proc. SPIE 1888.
Chance, B. \& Alfano, R. R. (eds) 1995 Optical tomography, photon migration, and spectroscopy of tissue and model media: theory, human studies, and instrumentation. Proc. SPIE 2389.

Chandrasekhar, R. 1950 Radiation transfer. Oxford: Clarendon Press.

Cope, M. \& Delpy, D. T. 1988 System for the long-term measurement of cerebral blood and tissue oxygenation on newborn infants by near infrared transillumination. Med. Biol. Engng Comput. May, 289-294.

Delpy, D. T., Cope, M., van der Zee, P., Arridge, S., Wray, S. \& Wyatt, J. 1988 Estimation of optical pathlength through tissue from direct time of flight measurement. Phys. Med. Biol. 33, 1433-1442.

den Outer, P. N., Nieuwenhuizen, Th. M. \& Langendijk, A. 1993 Location of objects in multiple-scattering media. $J$. Opt. Soc. Am. A 10, 1209-1218.

de Oliveira, C. R. E. 1986 An arbitrary geometry finite element method for multigroup neutron transport with anisotropic scattering. Prog. Nucl. Energy 18, 227-236.

Duderstadt, J. J. \& Hamilton, L. J. 1976 Nuclear reactor analysis. New York: John Wiley \& Sons.

Eason, G., Veitch, A., Nisbet, R. \& Turnbull, F. 1978 The theory of the backscattering of light by blood. J. Phys. D 11, 1463-1479.

Feng, S., Zeng, F.-A. \& Chance, B. 1995 Photon migration in the presence of a single defect: a perturbation analysis. Appl. Opt. 34, 3826-3837.

Firbank, M., Arridge, S. R., Schweiger, M. \& Delpy, D. T. 1996 An investigation of light transport through scattering bodies with non-scattering regions. Phys. Med. Biol. 41, 767-783.

Graber, H. L., Chang, J., Lubowsky, J., Aronson, R. \& Barbour, R. L. 1993 Near-infrared absorption imaging of dense scattering media by steady-state diffusion tomography. Proc. SPIE 1888, 372-386.

Hackbush, W. 1980 Multigrid methods and applications. Berlin: Springer-Verlag.

Hebden, J. \& Arridge, S. R. 1997 Optical imaging in medicine I: experimental techniques. Phys. Med. Biol. 42 (5), 825-840.

Henderson, R. P. \& Webster, J. G. 1978 An impedance camera for spatially specific measurements of the thorax. IEEE Trans., Biomed. Eng. BME-25, 250-253.

Ishimaru, A. 1978 Wave propagation and scattering in random media, volume 1: single scattering and transport theory. New York: Academic Press.

Jiang, H., Paulsen, K. D., Osterberg, U. L., Pogue, B. W. \& Patterson, M. S. 1995 Simultaneous reconstruction of optical absorption and scattering maps in turbid media from near-infrared frequency-domain data. Opt. Lett. 20, 2128-2130.

Jöbsis, F. F. 1977 Noninvasive infrared monitoring of cerebral and myocardial oxygen sufficiency and circulatory parameters. Science 198, 1264-1267.

Kaltenbach, J.-M. \& Kaschke, M. 1993 Frequency and time-domain modelling of light transport in random media. In Medical optical tomography: functional imaging and monitoring (ed. G. Muller), pp. 65-86. Washington: SPIE.

Lewis, H.W. 1950 Multiple scattering in an infinite medium. Phys. Rev. 78, 526-529.

Natterer, F. 1986 The mathematics of computerised tomography. Stuttgart: Wiley-Teubner.

O’Leary, M. A., Boas, D. A., Chance, B. \& Yodh, A. G. 1995 Experimental images of heterogeneous turbid media by frequency domain diffusing photon tomography. Opt. Lett. 20, 426-428.

Patterson, M. S., Chance, B. \& Wilson, B. C. 1989 Time 
resolved reflectance and transmittance for the non-invasive measurement of tissue optical properties. Appl. Opt. 28, 2331-2336.

Patterson, M. S., Wilson, B. C. \& Wyman, D. R. 1992 The propagation of optical radiation in tissue. I. Models of radiation transport and their application. Lasers in Medical Science 6, 155-168.

Peraire, J., Vahdati, M., Morgan, K. \& Zienkiewicz, O. C. 1987 Adaptive remeshing for compressible flow computations. J. Comp. Phys. 72, 449-466.

Pogue, B. W., Patterson, M. S., Jiang, H. \& Paulsen, K. D. 1995 Initial assessment of a simple system for frequency domain diffuse optical tomography. Phys. Med. Biol. 40, 1709-1729.

Schotland, J. C., Haselgrove, J. C. \& Leigh, J. S. 1993 Photon hitting density. Appl. Opt. 32, 448-453.

Schweiger, M., Arridge, S. R. \& Delpy, D. T. 1993 a Application of the finite element method for the forward and inverse problem in optical tomography. J. Math. Imag. Vision 3, 263-283.

Schweiger, M., Arridge, S. R., Hiraoka, M. \& Delpy, D. T. 1995 The finite element method for the propagation of light in scattering media: boundary and source conditions. Med. Phys. 22, 1779-1792.

Schweiger, M., Arridge, S. R., Hiraoka, M., Firbank, M. \& Delpy, D. T. $1993 b$ Comparison of a finite element forward model with experimental phantom results: application to image reconstruction. Proc. SPIE 1888, 179-190.

Sevick, E. M., Burch, C. L., Frisoli, J. K. \& Lakowicz, J. R. 1994 Localization of absorbers in scattering media by use of frequency-domain measurements of time-dependent photon migration. Appl. Opt. 33, 3562-3571.

Singer, J. R., Grünbaum, F. A., Kohn, P. D. \& Zubelli, J. P. 1990 Image reconstruction of the interior of bodies that diffuse radiation. Science 248, 990-993. 